\title{
CÁLCULO DE LA RECARGA POTENCIAL DE ACUÍFEROS MEDIANTE UN BALANCE HÍDRICO DE SUELOS
}

\author{
Gunther Schosinsky N. \\ Escuela Centroamericana de Geología, Universidad de Costa Rica, \\ Apdo. 214-2060, Costa Rica. \\ Correo-e: gschosin@cariari.ucr.ac.cr
}

(Recibido: 13/11/06; aceptado: 22/01/07)

\begin{abstract}
In this study, an analysis system has been developed, with the purpose to determine the recharge using a soil water balance. One of the advantages of this methodology is that it considers several variables that have influence in the recharge, such as: monthly rainfall, rainfall retention, soil infiltration capacity, soil characteristics, vegetation coverage, rooting depth, real evapotranspiration and slope gradient. This methodology has been widely used in ground water investigations. The error produced by the described methodology, is usually less than eighty percent.

Key words: Potential recharge, aquifers, water balance, soils.

RESUMEN: En este estudio se ha desarrollado una metodología y un modelo, con el propósito de determinar la recarga potencial al acuífero mediante un balance de suelos. Una de las ventajas de dicha metodología es que considera varias variables que influyen en la recarga de los acuíferos, tales como: la precipitación mensual, la retención pluvial, la capacidad de infiltración de los suelos y sus características, la cobertura vegetal, profundidad de raíces, evapotranspiración real, uso del suelo y pendiente del terreno. La metodología descrita ha sido bastante utilizada, generando un error de recarga menor del ochenta por ciento, en los casos que se ha podido comprobar, en forma confiable, los valores de dicha recarga.

Palabras clave: Recarga potencial, acuíferos, balance hídrico, suelos.
\end{abstract}




\section{INTRODUCCIÓN}

La necesidad de conocer el potencial de aguas subterráneas para ser explotadas mediante pozos, conlleva a estimar la recarga de los acuíferos en proyectos de evaluación de las aguas subterráneas, lo que ha hecho que en Costa Rica se realicen estudios tendientes a establecer dicha recarga con base en la distribución de precipitación y coeficientes de infiltración en los suelos del país.

Para evaluar la infiltración de lluvia que penetra al suelo en una zona, se determinan: la precipitación mensual de la zona, los diferentes valores de infiltración básica de los suelos, la cobertura vegetal del suelo y su pendiente. Determinados los valores anteriormente mencionados, se puede evaluar la infiltración mediante la ecuación presentada por Schosinsky \& Losilla, (2000).

Conocida la infiltración, se puede realizar un balance de suelos para estimar el agua que queda libre para recargar el acuífero que se encuentra debajo del suelo analizado. Para poder realizar el balance, se determinan: la infiltración de lluvia que penetra al suelo, la cobertura vegetal del suelo, la profundidad de las raíces extractoras del agua, la capacidad de campo, el punto de marchitez del suelo, la evapotranspiración potencial y la humedad del suelo al inicio del análisis.

El estudio del balance de suelos se basa en el principio de la conservación de la materia. $\mathrm{O}$ sea, el agua que entra a un suelo, es igual al agua que se almacena en el suelo, más el agua que sale de él. Las entradas son debidas a la infiltración del agua hacia el suelo, y las salidas se deben a la evapotranspiración de las plantas, más la descarga de los acuíferos.

\section{BALANCE DE SUELOS}

El potencial de las aguas subterráneas de un acuífero, representa la máxima cantidad de agua a sustraer del acuífero, para que no sea sobreexplotado. Dicho potencial se estima mediante la recarga al acuífero, que se determina conociendo en primer lugar, la fracción de lluvia que es interceptada por el follaje. En segundo lugar, se requiere conocer la infiltración del agua de lluvia hacia el suelo, generada por la precipitación que llega a su superficie. En tercer lugar, se debe realizar un balance de suelos, que nos permita estimar el agua que drena del suelo hacia el acuífero, que se encuentra ubicado debajo del suelo.

\section{Fracción de lluvia interceptada por el follaje}

Lluvias menores de $5 \mathrm{~mm}$ mensuales, no van a generar infiltración ya que se considera que en un mes con lluvia, al menos $5 \mathrm{~mm}$ son retenidos por el follaje sin llegar al suelo (Schosinsky \& Losilla, 2000). Además, se considera que la retención de la lluvia en follajes, es del 12\% (Butler, 1957) de la precipitación mensual. Sin embargo, en bosques muy densos, la retención de la lluvia se considera en un 20\% (Linsley et al., 1958). Definiremos como coeficiente de follaje (Cfo) el porcentaje de la lluvia mensual que es retenida en el follaje, expresado en tanto por uno. Para el $12 \%$ de retención Cfo $=0,12$. Para el $20 \%$ de retención Cfo $=0,20$.

Para calcular la retención de lluvia mensual interceptada por el follaje (Ret), se aplicará la siguiente ecuación:

Ecuación para el cálculo de la retención mensual de lluvia por follaje.

Si P es menor o igual a $5 \mathrm{~mm} / \mathrm{mes}$, Ret $=\mathrm{P}$.

Si el producto $(\mathrm{P})(\mathrm{Cfo})$ es mayor o igual de 5 $\mathrm{mm} / \mathrm{mes}$, Ret $=(\mathrm{P})(\mathrm{Cfo})$.

Si $\mathrm{P}$ es mayor de $5 \mathrm{~mm} / \mathrm{mes}$ y el producto $(\mathrm{P})(\mathrm{Cfo})$ menor de 5 , Ret $=5$.

Donde:

$\mathrm{P}=$ Precipitación mensual del mes [mm/mes].

Ret $=$ Retención de lluvia en el follaje $[\mathrm{mm} / \mathrm{mes}]$.

Cfo $=$ Coeficiente de retención del follaje, para bosques muy densos $\mathrm{Cfo}=0,20$, otros $\mathrm{Cfo}=$ 0,12 [adimensional]. 
Ejemplo 1 - En un bosque muy denso llueve $3 \mathrm{~mm}$ en el mes de abril, entonces la lluvia retenida en el follaje es:

P es menor de $5 \mathrm{~mm}$, luego, de ecuación (1), Ret $=3 \mathrm{~mm}$

Ejemplo 2- En una zona de cultivo y bosque, llueve $100 \mathrm{~mm} / \mathrm{mes}$, ¿cuál es la lluvia retenida en el follaje? P es mayor de $5 \mathrm{~mm}$, Cfo $=0,12$

$(\mathrm{P})(\mathrm{Cfo})=100 * 0,12=12$ es mayor de 5 , de ecuación $(1)$, Ret $=100 * 0,12=12 \mathrm{~mm} / \mathrm{mes}$

Ejemplo 3 - En noviembre, en una zona boscosa no densa, la precipitación fue de $35 \mathrm{~mm}$. La lluvia retenida en el follaje es entonces: $\mathrm{P}$ es mayor de $5 \mathrm{~mm}, \mathrm{Cfo}=0,12$

$(\mathrm{P})(\mathrm{Cfo})=35^{*} 0,12=4,2$ es menor de 5 , de ecuación (1) Ret $=5 \mathrm{~mm} / \mathrm{mes}$

\section{Infiltración}

Uno de los factores que más influyen en la infiltración de la lluvia en el suelo, es el coeficiente de infiltración debido a la textura del suelo ( $\mathrm{Kfc}$ ), que está dado tentativamente por la siguiente ecuación (Schosinsky \& Losilla, 2000):

$$
\mathrm{Kfc}=0,267 \ln (\mathrm{fc})-0,000154 \mathrm{fc}-0,723
$$

\section{Donde:}

Kfc [adimensional $]=$ Coeficiente de infiltración (fracción que infiltra por textura del suelo);

fc $[\mathrm{mm} /$ día $]$ = Infiltración básica del suelo.

Para aplicar esta ecuación, el rango de fc ha de encontrarse entre 16 a $1568 \mathrm{~mm} /$ día. Para valores de $\mathrm{fc}$ menores a $16 \mathrm{~mm} / \mathrm{día}, \mathrm{Kfc}=0,0148 \mathrm{fc} / 16$. Para valores de fc mayor a $1568 \mathrm{~mm} /$ día, $\mathrm{Kfc}=1$. Con las observaciones mencionadas en el párrafo anterior, el cálculo del coeficiente de infiltración por textura del suelo, se representa en la siguiente ecuación:

Ecuación para calcular el coeficiente de infiltración por textura de suelo (Kfc)

$\mathrm{Si}$ fc se encuentra entre 16 y $1568 \mathrm{~mm} / \mathrm{día}$ $\mathrm{Kfc}=0,267 \ln (\mathrm{fc})-0,000154 \mathrm{fc}-0,723$

$\mathrm{Si}$ fc es menor a $16 \mathrm{~mm} /$ día $\mathrm{Kfc}=0,0148 \mathrm{fc} / 16$

$\mathrm{Si}$ fc es mayor de $1568 \mathrm{~mm} /$ día Kfc $=1$

El valor de fc corresponde a la permeabilidad del suelo saturado, en los primeros 30 centímetros de profundidad, por considerar que este es el espesor que está en contacto directo con el agua de lluvia. Dicho valor se obtiene en el campo, con la prueba de anillos aplicada en la superficie del terreno. También se puede obtener con el permeámetro de Guelph o con la prueba de Porchet, ambas aplicadas a una profundidad no mayor de $30 \mathrm{~cm}$. Sin embargo, pueden existir diferencias entre los métodos utilizados.

El valor de Kfc, fue derivado para los valores de lluvia mensual. Por lo tanto, la fracción que infiltra debido a la textura del suelo, nos permite obtener la infiltración mensual debido a este concepto. Además del coeficiente de infiltración debido a la textura del suelo, influye la pendiente del terreno y la vegetación. Estos coeficientes, vienen a conformar el coeficiente de infiltración del suelo (Ci), basado en la siguiente ecuación (ONU, 1972):

Ecuación para el cálculo del coeficiente de infiltración del suelo

Si $\mathrm{Kp}+\mathrm{Kv}+\mathrm{Kfc}$ es mayor de $1, \mathrm{Ci}=1$.

$\mathrm{Si} \mathrm{Kp}+\mathrm{Kv}+\mathrm{Kfc}$ es menor o igual a 1 , entonces $\mathrm{Ci}=\mathrm{Kp}+\mathrm{Kv}+\mathrm{Kfc}$.

Donde:

$\mathrm{Ci}=$ Coeficiente de infiltración [adimensional].

$\mathrm{Kp}=$ Fracción que infiltra por efecto de pendiente [adimensional] (Cuadro 1).

$\mathrm{Kv}=$ Fracción que infiltra por efecto de cobertura vegetal [adimensional] (Cuadro 1).

$\mathrm{Kfc}=$ Fracción que infiltra por textura del suelo [adimensional] (ecuación 2).

El coeficiente de infiltración es el factor por el cual hay que multiplicar la precipitación mensual para obtener el agua que se infiltra mensualmente hacia el suelo. En una zona dada, entre menor sea la pendiente del terreno y mayor sea su cobertura vegetal, la velocidad de escurrimiento se retrasa, generando una mayor infiltración. Los valores sugeridos de estos componentes, que 
conforman el coeficiente de infiltración, se muestran en el Cuadro 1.

\section{Cálculo de infiltración pluvial mensual}

En el cálculo de la precipitación que infiltra mensualmente, se han de considerar los siguientes factores: la precipitación mensual, la retención pluvial mensual en el follaje (ecuación 1) y el coeficiente de infiltración (ecuación 3).

El cálculo de la precipitación que infiltra mensualmente $(\mathrm{Pi})$ al suelo, está dado por la siguiente ecuación:

$$
\mathrm{Pi}=(\mathrm{Ci})(\mathrm{P}-\mathrm{Ret})
$$

Donde:

$\mathrm{Pi}=$ Precipitación que infiltra mensualmente al suelo en $[\mathrm{mm} / \mathrm{mes}]$.

$\mathrm{Ci}=$ Coeficiente de infiltración [adimensional] (ecuación 3)

\section{Cuadro 1}

Componentes del coeficiente de infiltración (Schosinsky \& Losilla, 2000).

\begin{tabular}{lcc}
\hline Por pendiente: & $\begin{array}{c}\text { Pendiente } \\
\text { Muy plana }\end{array}$ & $\mathrm{Kp}$ \\
Plana & $0,02 \%-0,06 \%$ & 0,30 \\
Algo plana & $1 \%-2 \%$ & 0,20 \\
Promedio & $2 \%-7 \%$ & 0,15 \\
Fuerte & mayor de $7 \%$ & 0,10 \\
\hline & 0,06 \\
\hline Por cobertura vegetal: & \\
Cobertura con zacate menos del 50\% & $\mathrm{Kv}$ \\
Terrenos cultivados & 0,09 \\
Cobertura con pastizal & 0,10 \\
Bosques & 0,18 \\
Cobertura con zacate más del $75 \%$ & 0,20 \\
\hline
\end{tabular}

$\mathrm{P}=$ Precipitación mensual en $[\mathrm{mm} / \mathrm{mes}]$ (dato meteorológico).

Ret $=$ Retención de lluvia mensual por follaje en [mm/mes] (ecuación 1).

En ningún caso el coeficiente de infiltración (Ci) ha de ser mayor de 1 , si así fuese, se le asigna a $\mathrm{Ci}$ el valor de 1.

\section{Escorrentía superficial}

La escorrentía superficial generada por la lluvia mensual, corresponde a la precipitación mensual menos la retención de lluvia en el follaje menos la infiltración. La escorrentía mensual se calcula con la siguiente ecuación

$$
\mathrm{ESC}=\mathrm{P}-\mathrm{Ret}-\mathrm{Pi}
$$

$\mathrm{ESC}=$ Escorrentía superficial en $\mathrm{mm} / \mathrm{mes}$.

$\mathrm{P}=$ Precipitación en $\mathrm{mm} / \mathrm{mes}$ (dato meteorológico).

Ret $=$ Retención de lluvia mensual por follaje en $\mathrm{mm} / \mathrm{mes}$ (ecuación 1).

Pi $=$ Precipitación que infiltra mensualmente al suelo en $\mathrm{mm} / \mathrm{mes}$ (ecuación 4).

\section{Balance del suelo}

Para el balance del suelo, en primera instancia se requiere la infiltración mensual al suelo, generada por la lluvia. Dicho cálculo se obtiene de acuerdo con el apartado de "Infiltración" descrito anteriormente. Posteriormente, es necesario conocer la capacidad de campo y punto de marchitez del suelo. Estos valores, se obtienen directamente del laboratorio de suelos o se estiman mediante el Cuadro 2. También es necesario conocer la profundidad aproximada de las raíces extractoras de agua, en la zona donde se ha de realizar el balance. O sea, el balance se realizará en un prisma rectangular, que tiene en la cara superior un cuadrado de 1 metro de lado y de profundidad, la de las raíces, con capacidad 
Ejemplo 4 - La precipitación en el mes de octubre es de $200 \mathrm{~mm}$. La infiltración básica del suelo (permeabilidad de suelo a 24 horas de saturación) es de $85 \mathrm{~mm} / \mathrm{d}$. La zona se encuentra con cobertura de $50 \%$ zacate y $50 \%$ de bosque, con pendientes superiores al 7\%. La infiltración en octubre sería.

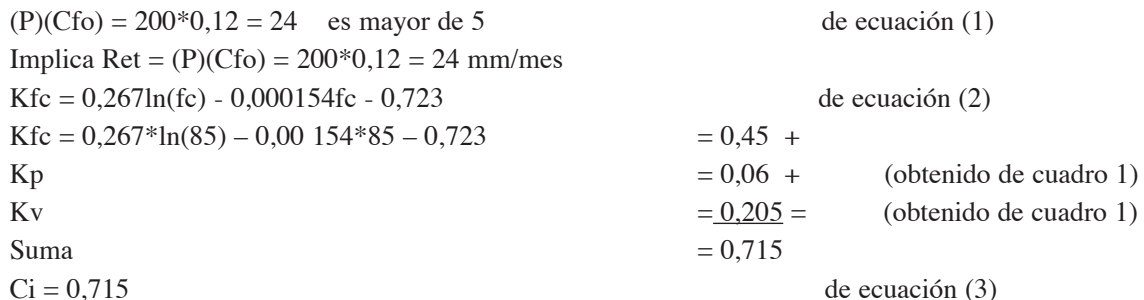

El valor de Ci es menor de 1 y la precipitación de 200 mm/mes, por lo tanto, la infiltración para el mes de octubre será:

$\mathrm{Pi}=(\mathrm{Ci})(\mathrm{P}-\mathrm{Ret})$

de ecuación (4)

$\mathrm{Pi}=0,715 *(200-24)=125,84 \mathrm{~mm} / \mathrm{mes}$

Ejemplo 5 - La precipitación del mes de mayo es de $100 \mathrm{~mm}$. La infiltración básica del suelo es de $200 \mathrm{~mm} / \mathrm{d}$. La zona se encuentra con cobertura de $80 \%$ de zacate, con pendientes menores del 1\%. La infiltración en mayo sería:

$(\mathrm{P})(\mathrm{Cfo})=100 * 0,12=12$ que es mayor de $5 \quad$ de ecuación $(1)$

Implica Ret $=(\mathrm{P})(\mathrm{Cfo})=100 * 0,12=12 \mathrm{~mm} / \mathrm{mes}$

$\mathrm{Kfc}=0,267 \ln (\mathrm{fc})-0,000154 \mathrm{fc}-0,723 \quad$ de ecuación (2)

$\mathrm{Kfc}=0,267 * \ln (200)-0,000154 * 200-0,723=0,66+$

$\mathrm{Kp} \quad=0,20+\quad$ (obtenido de cuadro 1)

$\mathrm{Kv} \quad=\underline{0.21}=\quad$ (obtenido de cuadro 1 )

Suma $\quad=1,07$

$\mathrm{Ci}=1 \quad$ de ecuación (3)

El valor de $\mathrm{Ci}$ es mayor de 1 por lo que se utiliza $\mathrm{Ci}=1$, por lo tanto la infiltración para el mes de mayo será:

$\mathrm{Pi}=(\mathrm{Ci})(\mathrm{P}-\mathrm{Ret}) \quad$ de ecuación (4)

$\mathrm{Pi}=1 *(100-12)=88 \mathrm{~mm} / \mathrm{mes}$

Ejemplo 6 - La precipitación en abril es de $38 \mathrm{~mm}$. La infiltración básica del suelo es de $85 \mathrm{~mm} / \mathrm{d}$. La zona se encuentra con cobertura de pastizal, con pendientes del 6\%. La infiltración en abril sería:

$(\mathrm{P})(\mathrm{Cfo})=38 * 0,12=4,56$ es menor de $5 \quad$ de ecuación (1)

Implica Ret $=5 \mathrm{~mm} / \mathrm{mes}$

$\mathrm{Kfc}=0,267 \ln (\mathrm{fc})-0,000154 \mathrm{fc}-0,723$

$\mathrm{Kfc}=0,267 * \ln (85)-0,00154 * 85-0,723 \quad=0,45+$

$\mathrm{Kp}$

$\mathrm{Kv}$

$=0,10+\quad$ (obtenido de cuadro 1$)$

Suma

(obtenido de cuadro 1)

$\mathrm{Ci}=0,73$

El valor de Ci es menor de 1, por lo tanto la infiltración para el mes de abril sería:

$\mathrm{Pi}=\mathrm{Ci}(\mathrm{P}-\mathrm{Ret})=0,73 *(38-5)=24,09 \mathrm{~mm} / \mathrm{mes}$

Ejemplo 7 - La precipitación del mes de febrero es de $4 \mathrm{~mm}$. La infiltración básica del suelo es $200 \mathrm{~mm} / \mathrm{d}$. La zona se encuentra con cobertura de $80 \%$ zacate, con pendientes menores del 1\%. La infiltración para el mes de febrero sería:

$\mathrm{P}<5 \mathrm{~mm} / \mathrm{mes}$

Implica Ret $=\mathrm{P} \quad$ Ret $=4 \mathrm{~mm} / \mathrm{mes}$ de ecuación (1)

$\mathrm{Pi}=(\mathrm{Ci})(\mathrm{P}-\mathrm{Ret}) \quad$ de ecuación (4)

$\mathrm{Pi}=\mathrm{Ci}^{*}(4-4)=0 \mathrm{~mm} / \mathrm{mes}$

Al ser la precipitación de febrero menor de $5 \mathrm{~mm}$, la precipitación es interceptada por el follaje, por lo tanto no existe infiltración en el mes de febrero. 


\section{Cuadro 2}

Punto de marchitez permanente y capacidad de campo en porcentaje por peso de suelo seco de diferentes texturas de suelos (Grassi, 1976).

\begin{tabular}{c|ccc}
\hline \multirow{2}{*}{ TEXTURA DEL SUELO } & \multicolumn{3}{c}{ Porcentaje por peso de suelo seco } \\
\cline { 2 - 4 } & PMP\% & CC\% & Densidad aparente $\left(\mathrm{g} / \mathrm{cm}^{3}\right)$ \\
\hline Arenoso & $2-6$ & $6-12$ & $1,55-1,80$ \\
Franco-arenoso & $4-8$ & $10-18$ & $1,40-1,60$ \\
Franco & $8-12$ & $18-26$ & $1,35-1,50$ \\
Franco- arcilloso & $11-15$ & $23-31$ & $1,30-1,40$ \\
Arcillo-arenoso & $13-17$ & $27-31$ & $1,25-1,35$ \\
Arcilloso & $15-19$ & $31-39$ & $1,20-1,30$ \\
\hline
\end{tabular}

de absorción del agua en el suelo. El Cuadro 3 muestra la profundidad mencionada de raíces de algunas plantas.

La forma natural de extracción de agua del suelo es mediante la transpiración de las plantas. Dicha extracción se realiza mediante las raíces; por lo tanto, la extracción de agua se realizará en una franja de suelo que tiene una profundidad igual a la mostrada en el Cuadro 3.

La máxima humedad que puede tener un suelo que no se encuentre saturado, es igual a la capacidad de campo, es entonces cuando la planta tiene la máxima capacidad de transpiración. La mínima humedad que puede tener un suelo es aproximadamente igual al punto de marchitez, pues con humedades menores la planta muere. De lo anterior se deduce que un suelo, no saturado, a profundidades mayores que la profundidad de raíces se encuentra a capacidad de campo.

\section{Evapotranspiración}

La evapotranspiración en una zona de cultivo se define como la transpiración de la planta, cuando el suelo se encuentra a capacidad de campo, más la evaporación del suelo. La mayor capacidad de evapotranspiración de un cultivo es cuando el suelo se encuentra a capacidad de campo. Sin embargo, cuando la humedad del suelo es menor que la capacidad de campo, las hojas de las plantas van cerrando los estomas, con el propósito de transpirar menos y así economizar el agua.
Cuando la humedad del suelo llega al punto de marchitez permanente, la planta no transpira y muere (Heras, 1972). En este estudio, asumiremos que la evapotranspiración potencial real va a ser proporcional a la humedad del suelo, comparada con la diferencia de humedad entre la capacidad de campo y el punto de marchitez. Esto es, si una planta a capacidad de campo evapotranspira $3 \mathrm{~mm}$ por día, siendo la capacidad de campo del suelo $12 \%$, el punto de marchitez permanente de $2 \%$ y el suelo se encuentra a una humedad de $6 \%$, la evapotranspiración potencial real (ETPR) de la planta será de:

$$
\begin{aligned}
& \mathrm{ETPR}=(\mathrm{HS}-\mathrm{PM})(\mathrm{ET}) /(\mathrm{CC}-\mathrm{PM}) \\
& \mathrm{ETPR}=[(6-2) /(12-2)] * 3=1,2 \mathrm{~mm} / \mathrm{d}
\end{aligned}
$$

Cuadro 3

Profundidad de raíces de diferentes cultivos (Grassi, 1976).

\begin{tabular}{cc}
\hline \multicolumn{2}{c}{ PROFUNDIDAD DE RAÍCES } \\
\hline Cultivo & Metros \\
\hline Alfalfa (pastos) & $1-2$ \\
Algodón & $1-1,7$ \\
Banano & $0,5-0,8$ \\
Caña de azúcar & $1,20-2$ \\
Frijol & $0,5-0,7$ \\
Cebolla & $0,3-0,5$ \\
Cítricos & $1,20-2,0$ \\
Zacate & $0,3-0,5$ \\
Bosques & $2,0-3,0$ \\
\hline
\end{tabular}


Ejemplo 8 - La temperatura media mensual para el mes de julio es de $25^{\circ} \mathrm{C}$, determine la evapotranspiración mensual, para dicho mes, utilizando Blaney Criddle, sabiendo que la zona se encuentra en latitud $10^{\circ}$ norte.

Donde:

ETPR $[\mathrm{mm} /$ día $]=$ Evapotranspiración potencial real

HS $[\%]=$ Humedad del suelo

ET $[\mathrm{mm} /$ día $]=$ Evapotranspiración de la planta a capacidad de campo

$\mathrm{CC}[\%]=$ Capacidad de campo

PM [\%] = Punto de marchitez permanente

Cada planta tiene una evapotranspiración diferente y va a depender de la temperatura de ambiente, humedad relativa, radiación solar, velocidad de viento y grado de desarrollo de la planta (Heras, 1972).

En una cuenca o en una zona arbitraria de la cuenca, es casi imposible determinar la evapotranspiración de la vegetación, debido a la gran variedad de vegetación y grado de desarrollo de las plantas existentes en una cuenca o en una zona de la misma. Por este motivo, es conveniente asumir una evapotranspiración, que se estime como promedio de la cuenca. Esta evapotranspiración promedio de la cuenca, la asumiremos que es igual la evapotranspiración potencial (ETP).

Para el cálculo de la evapotranspiración potencial, existen una serie de ecuaciones, algunas de ellas requieren una serie de datos, que pocas estaciones meteorológicas los tienen. Por este motivo, se utilizará la ecuación de Blaney \& Criddle (ONU, 1972):

$$
\operatorname{ETP}(\mathrm{mm} / \mathrm{mes})=(8,10+0,46 \mathrm{~T}) \text { Ps }
$$

Donde:

ETP $=$ Evapotranspiración potencial en [ $\mathrm{mm} / \mathrm{mes}]$

$\mathrm{T}=$ Temperatura media mensual en [grados centígrados] (dato meteorológico)

Ps = Porcentaje de horas de luz solar mensual, con respecto al año [\%]

(Cuadro 4)
Existen tablas que tienen los porcentajes de luz solar para las diferentes latitudes, tanto del hemisferio norte como del sur. En el Cuadro 4 se presentan dichos valores para la latitud $10^{\circ}$ del hemisferio norte, por ser dicha latitud, en la que se ubica Costa Rica.

\section{Recarga al acuífero}

Para ser consistente con las unidades de los parámetros que se utilizaran en adelante, tal como capacidad de campo, punto de marchitez, humedad del suelo, precipitación y evapotranspiración se utilizarán unidades en milímetros.

Para poder convertir la humedad del suelo, punto de marchitez y capacidad de campo a milímetros, los pasaremos de porcentaje por peso de suelo seco, tal como aparecen en tablas o laboratorio de suelos, a porcentaje por volumen mediante la siguiente ecuación:

$\%$ por volumen $=(\%$ por peso de suelo seco $)$ (densidad aparente) (8)

Cuando ocurre la precipitación, el agua que infiltra se acumula en los poros del suelo, para llevarlo a capacidad de campo, que es la máxima acumulación de agua que puede tener un suelo no saturado. Posteriormente, una vez terminado el aguacero, se lleva a cabo la evapotranspiración, tomando las raíces el agua que se encuentra en los poros del suelo. Si la cantidad de infiltración de la lluvia es suficiente para llevar al suelo a capacidad de campo y llenar la necesidad de evapotranspiración, el sobrante del agua que infiltra, percola para recargar al acuífero.

Al siguiente coeficiente, contenido en la ecuación 6, lo designaremos coeficiente de

Cuadro 4

Porcentaje de horas de sol mensual, respecto al año, en la latitud $10^{\circ}$ Norte (Calvo, 1999)

\begin{tabular}{|c|c|c|c|c|c|c|c|c|c|c|c|c|}
\hline Mes & E & $\mathrm{F}$ & M & A & M & $\mathrm{J}$ & $\mathrm{J}$ & A & S & $\mathrm{O}$ & $\mathrm{N}$ & $\mathrm{D}$ \\
\hline$\%$ & 8,13 & 7,47 & 8,45 & 8,37 & 8,81 & 8,60 & 8,86 & 8,71 & 8,25 & 8,34 & 7,91 & 8,10 \\
\hline
\end{tabular}


Ejemplo 9 - Un suelo tiene una capacidad de campo de $8 \%$ por peso de suelo seco, y una densidad aparente de suelo seco de $1,4 \mathrm{~g} / \mathrm{cm}^{3}$ (obtenido de Cuadro 2), determine la capacidad de campo en porcentaje por volumen:

$\%$ de capacidad de campo por volumen $=8 * 1,4=11,2 \%$

La planta hará uso de la humedad del suelo, en una franja igual a la profundidad de raíces. Para convertir la humedad en $\%$ por volumen en milímetros de agua existentes en la franja de profundidad de raíces, se utiliza la siguiente ecuación:

Milímetros de agua $=(\%$ de humedad por volumen $)($ profundidad de raíces en $\mathrm{mm})$

Ejemplo 10 - Un suelo tiene una humedad de $10 \%$ por volumen (ecuación 8), la profundidad de raíces es de 1,5 metros (obtenida de Cuadro 3). Determine los milímetros de agua correspondientes a dicha humedad.

Milímetros de agua $=0,10 * 1,5 * 1000=150 \mathrm{~mm}$ con ecuación (9)

humedad (Ch): $\mathrm{Ch}=$ (humedad de suelo - punto de marchitez) / (capacidad de campo - punto de marchitez)

Al iniciar un mes cualquiera, el suelo tendrá una humedad inicial (HSi). Si no existiese evapotranspiración, la precipitación que infiltra (Pi) vendría a aumentar la humedad en el suelo, permitiendo una mayor evapotranspiración. Si no consideramos la evapotranspiración, el coeficiente de humedad, al final del mes, sería (C1):

$$
\mathrm{C} 1=(\mathrm{HSi}-\mathrm{PM}+\mathrm{Pi}) /(\mathrm{CC}-\mathrm{PM})
$$

Donde:

$\mathrm{C} 1=$ Coeficiente de humedad al final del mes antes de que ocurra la evapotranspiración.

Hsi = Humedad al inicio del mes, humedad de suelo inicial en $[\mathrm{mm}]$.

$\mathrm{PM}=$ Punto de marchitez en [mm].

$\mathrm{Pi}=$ Precipitación que infiltra en $[\mathrm{mm} / \mathrm{mes}]$.

$\mathrm{CC}=$ Capacidad de campo en [mm].

$\mathrm{Si}$ consideramos que ocurre la evapotranspiración, una vez ocurrida la infiltración, el coeficiente de humedad, al final del mes sería:

$$
\mathrm{C} 2=(\mathrm{HSi}-\mathrm{PM}+\mathrm{Pi}-\mathrm{ETR} 1) /(\mathrm{CC}-\mathrm{PM})
$$$$
\mathrm{ETR} 1=(\mathrm{C} 1)(\mathrm{ETP})
$$

Donde:

C2 = Coeficiente de humedad al final del mes, después de que ocurra la evapotranspiración.

ETR1 = Evapotranspiración potencial real $[\mathrm{mm} /$ mes], considera la humedad correspondiente al coeficiente $\mathrm{C} 1$.

$\mathrm{ETP}=$ Evapotranspiración potencial $[\mathrm{mm} / \mathrm{mes}]$.

El valor de $\mathrm{C} 1$ corresponde al coeficiente de humedad máximo, ya que considera la humedad del suelo al inicio del mes, más la infiltración de la lluvia, sin ocurrir la evapotranspiración. El valor de $\mathrm{C} 2$, corresponde al coeficiente de humedad mímino, ya que está calculado considerando la humedad del suelo anterior, restándole la evapotranspiración mensual, estimada con el coeficiente de humedad máximo, C1. Por lo tanto, el coeficiente $\mathrm{C} 2$ se aproxima al coeficiente de humedad al final del mes.

Como la infiltración y la evapotranspiración ocurre durante el mes, se estima que el coeficiente de humedad del mes corresponde al promedio de $\mathrm{C} 1$ y $\mathrm{C} 2$; o sea, $(\mathrm{C} 1+\mathrm{C} 2) / 2$; esto quiere decir que la evapotranspiración potencial real ocurrida en un mes dado es:

$$
\operatorname{ETPR}(\mathrm{mm} / \mathrm{mes})=((\mathrm{C} 1+\mathrm{C} 2) / 2) \operatorname{ETP}
$$

\section{Donde:}

ETPR $=$ Evapotranspiración real tentativa promedio, en una zona, ocurrida durante el mes [mm/mes]

$\mathrm{C} 1$ = Coeficiente de humedad máximo, sin considerar la evapotranspiración.

C2 = Coeficiente de humedad mínimo considerando evapotranspiración calculada con $\mathrm{C} 1$.

ETP = Evapotranspiración potencial [mm/mes]

Ninguno de los coeficientes de humedad, C1 y $\mathrm{C} 2$, pueden ser superiores a 1 , ni menores a 0 . En caso que $\mathrm{C} 1$ o $\mathrm{C} 2$, sea mayor de 1, se tomará igual a 1. Si $\mathrm{C} 1$ o $\mathrm{C} 2$ son negativos se tomarán con valor de 0 .

Definiremos como humedad disponible (HD), aquella humedad que pueden tomar las raíces de las plantas, para poder evapotranspirar. La humedad disponible está dada por la siguiente ecuación:

$$
\mathrm{HD}(\mathrm{mm} / \mathrm{mes})=\mathrm{HSi}+\mathrm{Pi}-\mathrm{PM}
$$


Donde:

$\mathrm{HD}=$ Humedad disponible $[\mathrm{mm} / \mathrm{mes}]$.

Hsi = Humedad de suelo inicial (al inicio del mes) $[\mathrm{mm}]$.

$\mathrm{Pi}=$ Precipitación que infiltra $[\mathrm{mm} / \mathrm{mes}]$.

$\mathrm{PM}=$ Punto de marchitez [mm].

Si la humedad disponible es menor que la ETPR de la ecuación (12), la planta no podrá evapotranspirar dicha cantidad, sino que evapotranspirará únicamente la humedad disponible, debido a que no hay suficiente humedad para evapotranspirar la cantidad de agua indicada en la ecuación (12). Sin embargo, si la humedad disponible (HD) es mayor que la cantidad de agua indicada en la ecuación (12), la planta evapotranspirará la cantidad expresada en dicha ecuación. Por lo tanto la evapotranspiración real será:

Ecuación para el cálculo de evapotranspiración real (ETR)

$\mathrm{Si}((\mathrm{C} 1+\mathrm{C} 2) / 2) \mathrm{ETP}$ es menor o igual a HD,

$\mathrm{ETR}(\mathrm{mm} / \mathrm{mes})=((\mathrm{C} 1+\mathrm{C} 2) / 2) \mathrm{ETP}$

$\mathrm{Si}((\mathrm{C} 1+\mathrm{C} 2) / 2) \mathrm{ETP}$ es mayor que $\mathrm{HD}$,

$\mathrm{ETR}(\mathrm{mm} / \mathrm{mes})=\mathrm{HD}$

Donde:

ETR = Evapotranspiración real promedio de la zona, ocurrida durante el mes [mm/mes].

$\mathrm{C} 1$ = Coeficiente de humedad máximo, sin considerar la evapotranspiración [adimensional].

C2 = Coeficiente de humedad mínimo considerando evapotranspiración calculada con C1, [adimensional].

ETP $=$ Evapotranspiración potencial $[\mathrm{mm} /$ mes].

$\mathrm{HD}=$ Humedad disponible $[\mathrm{mm} / \mathrm{mes}]$

Para poder realizar el cálculo de la recarga del acuífero, se requiere conocer la humedad del suelo al final del mes, humedad de suelo final (Hsf), la cual no puede ser mayor que la capacidad de campo y se obtiene con la siguiente ecuación:

Ecuación para el cálculo de HSf

$\mathrm{Si}(\mathrm{HD}+\mathrm{PM}-\mathrm{ETR})$ es menor que la capacidad de campo, HSf $=$ HD + PM - ETR

$\mathrm{Si}$ (HD + PM - ETR) es mayor o igual que la capacidad de campo, $\mathrm{HSf}=\mathrm{CC}$
La HSf en ningún momento puede ser mayor a la CC.

Donde:

$\mathrm{HSf}=$ Humedad del suelo final (final de mes) $[\mathrm{mm}]$.

$\mathrm{HD}=$ Humedad disponible $[\mathrm{mm} / \mathrm{mes}]$.

$\mathrm{PM}=$ Punto de marchitez $[\mathrm{mm}]$.

$\mathrm{ETR}=$ Evapotranspiración real $[\mathrm{mm} / \mathrm{mes}]$.

$\mathrm{CC}=$ Capacidad de campo [mm].

Además del cálculo de la ecuación para la humedad del suelo al final del mes, es necesario la humedad de suelo al inicio del mes, o sea, humedad inicial (HSi). La humedad inicial de un mes dado es la siguiente:

$\mathrm{HSi}=$ Es igual a la humedad de suelo final del mes anterior (HSf de ecuación 15)

$\mathrm{HSi}=$ Humedad del suelo inicial (inicio de mes) [mm].

HSf = Humedad del suelo final (final de mes) [mm].

\section{Cálculo de recarga potencial al acuífero}

La recarga al acuífero se lleva a cabo, si la cantidad de agua que infiltra es suficiente para llevar al suelo a capacidad de campo y además satisfacer la evapotranspiración de las plantas. El agua sobrante, una vez satisfecha la capacidad de campo y la evapotranspiración, es la que recarga al acuífero, la que se calcula con la siguiente ecuación.

$$
\mathrm{Rp}=\mathrm{Pi}+\mathrm{HSi}-\mathrm{HSf}-\mathrm{ETR}
$$

Donde:

$\mathrm{Rp}=$ Recarga potencial mensual en $\mathrm{mm} / \mathrm{mes}$.

$\mathrm{Pi}=$ Precipitación que infiltra en $\mathrm{mm} / \mathrm{mes}$.

HSf $=$ Humedad del suelo al final del mes en mm. ETR $=$ Evapotranspiración real en $\mathrm{mm} / \mathrm{mes}$.

\section{Otros términos de interés}

Existen otros términos de interés que pueden ser calculados, los que se describirán a continuación.

El déficit de capacidad de campo (DCC), es la humedad en mm que falta, para que la humedad 
Ejemplo 11 - Determine la evapotranspiración real, correspondiente al mes de junio, sabiendo que la capacidad de campo (CC) es $146 \mathrm{~mm}$, el punto de marchitez (PM) es de 94,9 mm, la humedad inicial (HSi) es de 104,91 mm, la precipitación que infiltra en el mes de junio (Pi) es de $83,25 \mathrm{~mm} /$ mes y la evapotranspiración potencial (ETP) es 159,26 $\mathrm{mm} / \mathrm{mes}$.

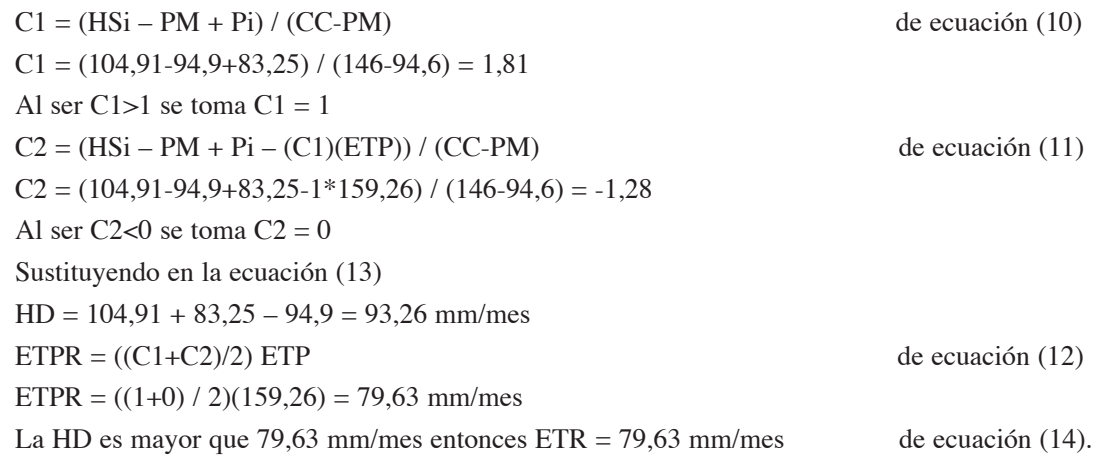

Ejemplo 12 - Determine la evapotranspiración real, correspondiente al mes de agosto, sabiendo que la capacidad de campo (CC) es $146 \mathrm{~mm}$, el punto de marchitez (PM) es 94,9 mm, la humedad inicial (HSi) es 94,9 mm, la precipitación que infiltra (Pi) es 184,54 mm/mes y la evapotranspiración potencial (ETP) es 163,97 mm/mes..

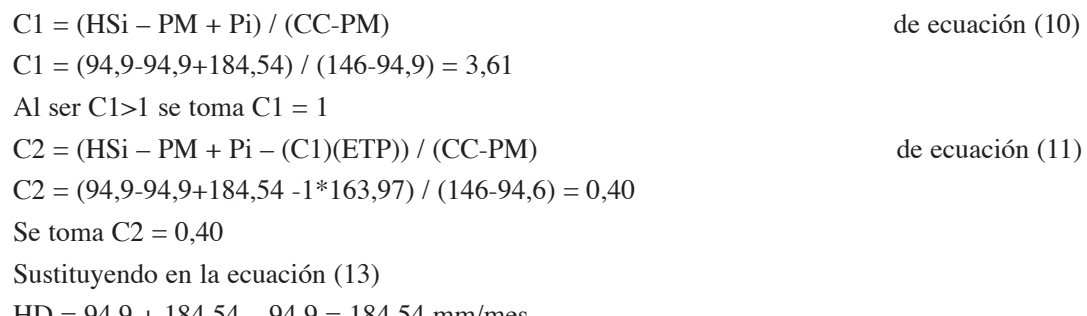

$\mathrm{HD}=94,9+184,54-94,9=184,54 \mathrm{~mm} / \mathrm{mes}$

Sustituyendo en la ecuación (12)

ETPR $=((1+0,40) / 2)(163,97)=114,78 \mathrm{~mm} / \mathrm{mes}$

La HD es mayor que la ETPR, entonces según la ecuación (14) ETR = 114,78 mm/mes.

Ejemplo 13 - Determine la evapotranspiración real, correspondiente al mes de julio, sabiendo que la capacidad de campo (CC) es de $146 \mathrm{~mm}$, el punto de marchitez (PM) es de 94,9 mm, la humedad inicial (HSi) es de 108,52 mm, la precipitación que infiltra $(\mathrm{Pi})$ es de 15,91 mm/mes y la evapotranspiración potencial (ETP) es de 161,85 mm/mes.

Sustituyendo en la ecuación (10)

$\mathrm{C} 1=(108,52-94,9+15,91) /(146-94,6)=0,576$

$\mathrm{A} 1$ ser $\mathrm{C} 1<1$ y $\mathrm{C} 1>0$ se toma $\mathrm{C} 1=0,576$

Sustituyendo en la ecuación (11)

$\mathrm{C} 2=(108,52-94,9+15,91-0,576 * 161,85) /(146-94,6)=-1,24$

Se toma $\mathrm{C} 2=0$.

Sustituyendo en la ecuación (13).

$\mathrm{HD}(\mathrm{mm} / \mathrm{mes})=108,52+15,91-94,9=29,53 \mathrm{~mm} / \mathrm{mes}$.

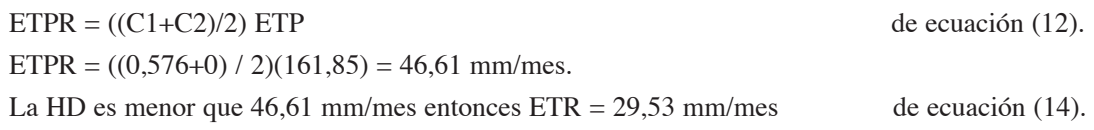


Ejemplo 14 - En el mes de julio, la precipitación que infiltra (Pi) es de 15,91 mm/mes, la humedad de suelo al inicio de julio (Hsi) es de 108,52 mm, la humedad disponible (HD) es de 29,53 mm/mes, el punto de marchitez (PM) es de 94,9 mm, la evapotranspiración real (ETR) es de 29,53 mm/mes y la capacidad de campo (CC) es de $146 \mathrm{~mm}$. Determine la recarga potencial al acuífero en el mes de julio.
$\mathrm{Rp}=\mathrm{Pi}+\mathrm{HSi}-\mathrm{HSf}-\mathrm{ETR}$
de ecuación (17)
$\mathrm{HSf}=\mathrm{HD}+\mathrm{PM}-\mathrm{ETR}$ si $(\mathrm{HD}+\mathrm{PM}-\mathrm{ETR})<\mathrm{CC}$; si es $>\mathrm{CC} \quad \mathrm{HSf}=\mathrm{CC}$
de ecuación (15)
$\mathrm{HD}+\mathrm{PM}-\mathrm{ETR}=$ 29,53+94,9-29,53 = 94,9 mm.
HD+PM-ETR no es mayor a la capacidad de campo, entonces HSf $=94,9 \mathrm{~mm}$ de ecuación (15)
$\mathrm{Rp}=15,91+108,52-94,9-29,53=0 \mathrm{~mm} / \mathrm{mes}$
de ecuación (17)

del suelo, alcance la capacidad de campo. Este déficit se refiere al final del mes.

$$
\mathrm{DCC}=\mathrm{CC}-\mathrm{HSf}
$$

Donde:

DCC $=$ Déficit de capacidad de campo, al final del mes $[\mathrm{mm}]$.

$\mathrm{CC}=$ Capacidad de campo en [mm].

$\mathrm{HSf}=$ Humedad del suelo al final del mes [mm].

La necesidad de riego (NR), se refiere a la cantidad de agua, mediante riego, que habría que agregar al suelo durante el mes, para mantener el suelo siempre a capacidad de campo. De tal forma que la planta pueda evapotranspirar sin necesidad de cerrar estomas. Esta es la humedad ideal, para obtener la máxima producción de un cultivo.

Como se mencionó anteriormente, la evapotranspiración promedio de una zona aleatoria en una cuenca, se asume con un valor igual al de la evapotranspiración potencial. La necesidad de riego, se estima con la siguiente ecuación:

$$
\mathrm{NR}=\mathrm{DCC}-\mathrm{ETR}+\mathrm{ETP}
$$

Donde:

$\mathrm{NR}=$ Necesidad de riego mensual [mm $/ \mathrm{mes}]$.

DDC $=$ Déficit de capacidad de campo, al final del mes [mm].

ETR $=$ Evapotranspiración real $[\mathrm{mm} / \mathrm{mes}]$.

ETP $=$ Evapotranspiración potencial $[\mathrm{mm} / \mathrm{mes}]$.

\section{RECARGA POTENCIAL ANUAL}

Para determinar la recarga potencial anual de los acuíferos, es necesario conocer la humedad inicial del suelo en un mes determinado. Generalmente, no se lleva un registro de dicha humedad para poderla determinar.

Ejemplo 15 - A continuación se dan los datos de la precipitación que infiltra mensualmente (Pi) y los valores de la evapotranspiración potencial (ETP). Determine el mes a seleccionar con una humedad inicial igual a la capacidad de campo.

\begin{tabular}{ccccccccccccc}
\hline Concepto & Ene & Feb & Mar & Abr & May & Jun & Jul & Ago & Sep & Oct & Nov & Dic \\
\hline Pi $(\mathrm{mm})$ & 0,0 & 0,0 & 0,0 & 0,0 & 101 & 83 & 16 & 185 & 152 & 192 & 41 & 0,0 \\
ETP $(\mathrm{mm})$ & 123 & 160 & 197 & 197 & 182 & 159 & 162 & 164 & 135 & 126 & 142 & 151 \\
\hline
\end{tabular}

Respuesta: el mes a seleccionar es noviembre. 


\section{Estimación de la humedad inicial en un mes seleccionado}

Dentro de las humedades conocidas, que se pueden encontrar en un suelo, están la capacidad de campo o el punto de marchitez. En el caso de Costa Rica, generalmente al final de la época de mayor precipitación, el suelo se encuentra a capacidad de campo. Uno de los criterios para seleccionar el mes, en el que vamos a considerar que el suelo se encuentra a capacidad de campo, es seleccionar meses consecutivos, en que la precipitación que infiltra mensualmente supere la evapotranspiración potencial mensual, asignando al mes siguiente, de los meses consecutivos seleccionados, una humedad inicial igual a la capacidad de campo.

Conocida la humedad inicial de un mes, podemos calcular la humedad final de dicho mes, la que será igual a la humedad inicial del mes siguiente y así sucesivamente. Al final del ciclo, la humedad final calculada para el último mes, ha de coincidir con la humedad inicial del mes de partida, o sea, la del mes con humedad conocida.

Como se indica en el ejemplo 15, los meses consecutivos en que la precipitación que infiltra, supera la evapotranspiración potencial son agosto, septiembre y octubre. Esto quiere decir, que al mes de noviembre se le asignará una humedad de suelo inicial, igual a la capacidad de campo. Con esta humedad, se pueden ir calculando las humedades finales e iniciales de los suelos, en los meses siguientes. Al cerrar el ciclo, el mes de octubre ha de tener una humedad final, igual a la capacidad de campo, lo que confirma, que el supuesto tomado ha sido correcto.

Puede darse el caso que exista solo un mes, en que se de la condición de que la precipitación que infiltra supere a la evapotranspiración potencial; en este caso, se asume la capacidad de campo como la humedad inicial del suelo, en el mes siguiente al mes que cumple con la condición mencionada y luego se verifica, mediante el cálculo de las humedades en los meses siguientes. De tal forma, que al cerrar el ciclo, el mes anterior al mes que se le asignó la humedad inicial igual a la capacidad de campo, tenga una humedad final, igual a la capacidad de campo.

\section{Cálculo anual de la recarga potencial y otros parámetros mencionados}

En el Cuadro 5 se muestra un cálculo anual de la recarga potencial y otros parámetros mencionados, tal como: necesidad de riego, humedad disponible, humedad al final de mes, déficit de capacidad de campo etc. Además se muestra la simbología de los términos utilizados. En dicho cuadro, los números en negrita, indican valores que se obtienen de datos del Instituto Meteorológico Nacional, tal como la precipitación y la temperatura mensual. Datos de campo tal como las pruebas de infiltración para obtener la permeabilidad del suelo y también se muestran datos de tablas publicadas contenidas en diferentes cuadros en este trabajo.

Se muestran también los valores calculados en números de color negro normal, indicando las ecuaciones utilizadas para dicho cálculo, las que están contenidas en el informe. Con el número de identificación, de las ecuaciones, cuadros y ejemplos, indicados en el Cuadro 5, se pueden realizar los cálculos para obtener la recarga potencial mensual. La recarga potencial anual, corresponde a la suma de las recargas potenciales mensuales.

\section{MODELO EN EXCEL PARA EL CÁLCULO DE RECARGA POTENCIAL}

Se ha realizado un modelo en una hoja electrónica de Excel (Cuadro 6), que realiza automáticamente los cálculos de las ecuaciones mostradas en el Cuadro 5. En el cuadro 5 únicamente se ingresan los números en negrita, los que indican valores que se obtienen de 


\section{Cuadro 5}

Cuadros y ecuaciones a utilizar para el cálculo anual de la recarga potencial de un acuífero en mm.

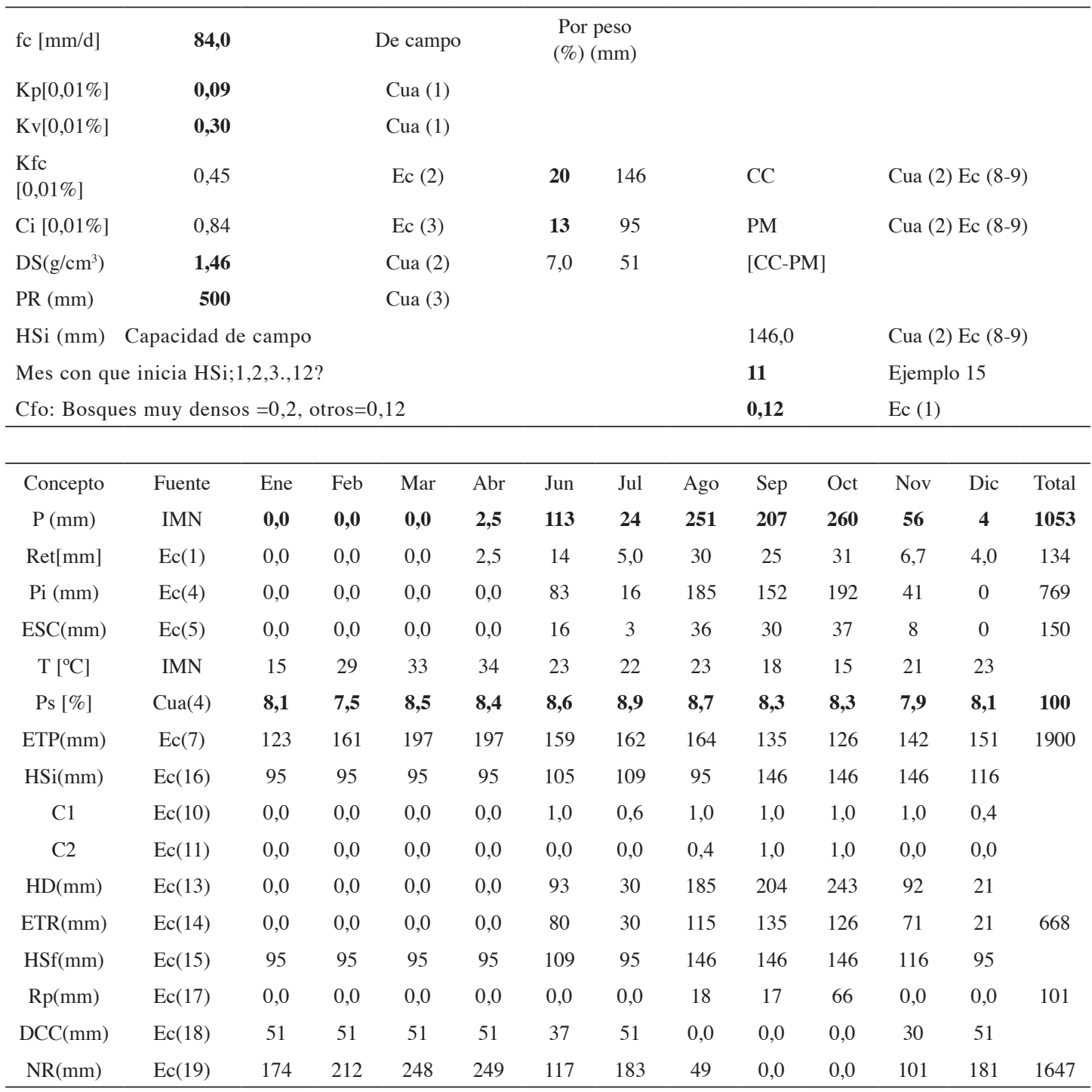

T=Temperatura; Ps=Porcentaje de horas sol; IMN= Instituto Meteorológico Nacional; Cua(4)=Cuadro 4; Ec (1)=Ecuación 1; fc =Capacidad de infiltración; $\mathrm{Ci}=$ Coeficiente de infiltración; $\mathrm{CC}=$ Capacidad de campo, $\mathrm{PM=Punto} \mathrm{de} \mathrm{marchitez;} \mathrm{PR=Profundidad}$ de raíces; $(\mathrm{CC}-\mathrm{PM})=$ Rango de agua disponible; DS=Densidad de suelo; $\mathrm{C} 1=$ Factor de ETP, por cierre de estomas, antes que ocurra ETR; $\mathrm{C} 2=$ Factor de ETP, por cierre de estomas, después que ocurre ETR; Kp=Factor por pendiente; Kv=Factor por vegetación; Kfc=Factor estimado con base a la prueba de infiltración; Cfo=Coficiente de retención pluvial en follajes; $\mathrm{P}=$ Precipitación media mensual; $\mathrm{Pi}=$ Precipitación que infiltra; $\mathrm{ESC}=$ Escorrentía superficial; $\mathrm{ETP}=\mathrm{Evapotranspiración} \mathrm{poten-}$ cial; ETR=Evapotranspiración real; HSi=Humedad de suelo inicial; HD=Humedad disponible; HSf=Humedad de suelo final; DCC=Déficit de capacidad de campo, Rp=Recarga potencial; NR=Necesidad de riego; Ret=Retención de lluvia. 
Cuadro 6

Modelo en Excel para el cálculo de recarga potencial de un acuífero en mm.

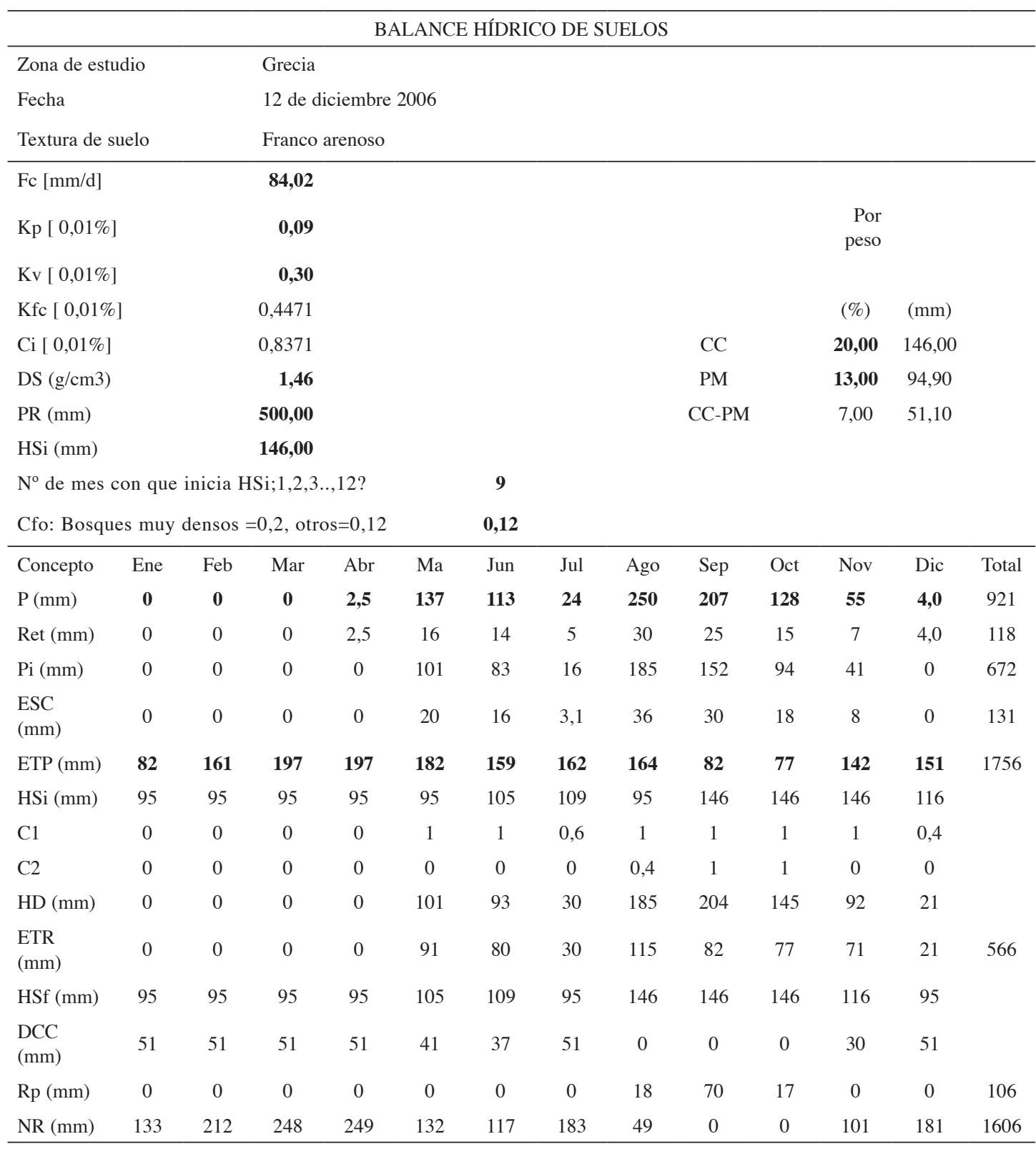

$\mathrm{Fc}=$ Capacidad de infiltración $; \mathrm{Ci}=$ Coeficiente de infiltración $; \mathrm{CC}=$ Capacidad de campo, $\mathrm{PM}=\mathrm{Punto}$ de marchitez; $\mathrm{PR}=\mathrm{Profundidad}$ de raíces; $(\mathrm{CC}-\mathrm{PM})=$ Rango de agua disponible; DS=Densidad de suelo; $\mathrm{C} 1=$ Factor de ETP, por cierre de estomas, antes que ocurra ETR; C2=Factor de ETP, por cierre de estomas, después que ocurre ETR; Kp=Factor por pendiente; Kv=Factor por vegetación; Kfc=Factor estimado con base a la prueba de infiltración; Cfo=Coeficiente de retención pluvial en follajes; $\mathrm{P}=$ Precipitación media mensual; $\mathrm{Pi}=$ Precipitación que infiltra; ESC=Escorrentía superficial; ETP=Evapotranspiración potencial; ETR=Evapotranspiración real; HSi=Humedad de suelo inicial; HD=Humedad disponible; HSf=Humedad de suelo final; DCC=Déficit de capacidad de campo, Rp=Recarga potencial; NR=Necesidad de riego; Ret=Retención de lluvia. 
datos del Instituto Meteorológico Nacional, tal como la precipitación, datos de campo, como las pruebas de infiltración para obtener la permeabilidad del suelo y también se muestran en negrita, datos obtenidos de tablas publicadas, que se muestran en diferentes cuadros en el informe. Con estos datos, el modelo calcula las ecuaciones requeridas para el cálculo de la recarga potencial.

La evapotranspiración potencial (ETP), se encuentra en negrita, no es calculada por el modelo. El motivo es dejar libre al usuario, de calcular dicha evapotranspiración por la ecuación que considere conveniente, ya que existen varias ecuaciones, como Blaney \& Criddle que requiere únicamente la temperatura media mensual; tanque de evaporación que no todas las estaciones meteorológicas lo tienen y la ecuación de Cristiansen, que requiere datos de humedad relativa, radiación solar y temperatura que no todas estaciones tienen dichos datos, etc.

La humedad incial (HSi) que generalmente usa la capacidad de campo (CC), se deja en libertad al usuario, en utilizar otra humedad inicial que no sea la capacidad de campo, por algún motivo especial.

\section{Cálculo de la recarga potencial anual en una cuenca}

Para el cálculo de recarga anual en una cuenca, es necesario dibujar la cuenca hidrogeológica a la que se le quiere calcular la recarga potencial anual en unidades de volumen. Una vez dibujada la cuenca, se requiere dibujar dentro de ella, las zonas con el mismo tipo de suelo o las zonas con el mismo tipo de geología, ya que una formación geológica forma un tipo determinado de suelo (Fig. 1).

\section{Polígonos de Thiessen}

Para realizar el cálculo de la recarga potencial, se seleccionan las áreas de diferente geología o clasificación de suelo, ya que estas se supone tienen una permeabilidad similar. En cada área conformada por una misma geología, se puede obtener un promedio de la permeabilidad, para mayor exactitud, es mejor realizar polígonos de Thiessen, para las diferentes pruebas de infiltración, dentro de la misma geología (Fig. 2).

$\mathrm{Al}$ existir dos estaciones meteorológicas, con diferentes valores de precipitación mensual dentro de la cuenca, se puede obtener un promedio de las precipitaciones para aplicar este valor a toda la cuenca. Sin embargo, para mayor exactitud, es conveniente realizar polígonos de Thiessen, para aplicar la precipitación correspondiente a cada polígono (Fig. 3).

Si combinamos los polígonos de las figuras 2 y 3, para obtener polígonos de tal forma que cada uno de ellos, tenga una precipitación determinada y una permeabilidad determinada (Fig. 4), obtendremos seis polígonos, en donde cada uno de ellos tendrá su precipitación y permeabilidad específica (Cuadro 7). El cuadro 7, muestra las características de precipitación y permeabilidad de cada uno de los polígonos indicados en la figura 4.

Para obtener la recarga potencial de la cuenca indicada en la figura 1, para cada uno de los polígonos del cuadro 7 , deberá realizarse los cálculos indicados en el cuadro 5 o cuadro 6 , para obtener la recarga potencial en cada uno de los polígonos. El volumen de agua que recarga al acuífero estará dado por la siguiente ecuación:

$$
\mathrm{V}=\mathrm{Rp} \mathrm{A}
$$

Donde:

$\mathrm{V}=$ Volumen de recarga $\left[\mathrm{m}^{3} / \mathrm{mes}\right.$ ó $\left.\mathrm{m}^{3} / \mathrm{año}\right]$.

$\mathrm{Rp}=$ Recarga potencial al acuífero [m/mes o m/año]. $\mathrm{A}=$ Área donde se genera la recarga potencial $\left[\mathrm{m}^{2}\right]$.

Cuadro 7

Precipitación y permeabilidad de cada uno de los polígonos indicados en la Fig. 4.

\begin{tabular}{ccc}
\hline Polígono & Precipitación & Permeabilidad \\
\hline Po1 & P2 & fc B1 \\
Po2 & P1 & fc B1 \\
Po3 & P1 & fc A1 \\
Po4 & P1 & fc A2 \\
Po5 & P2 & fc A2 \\
Po6 & P2 & fc A1 \\
\hline
\end{tabular}




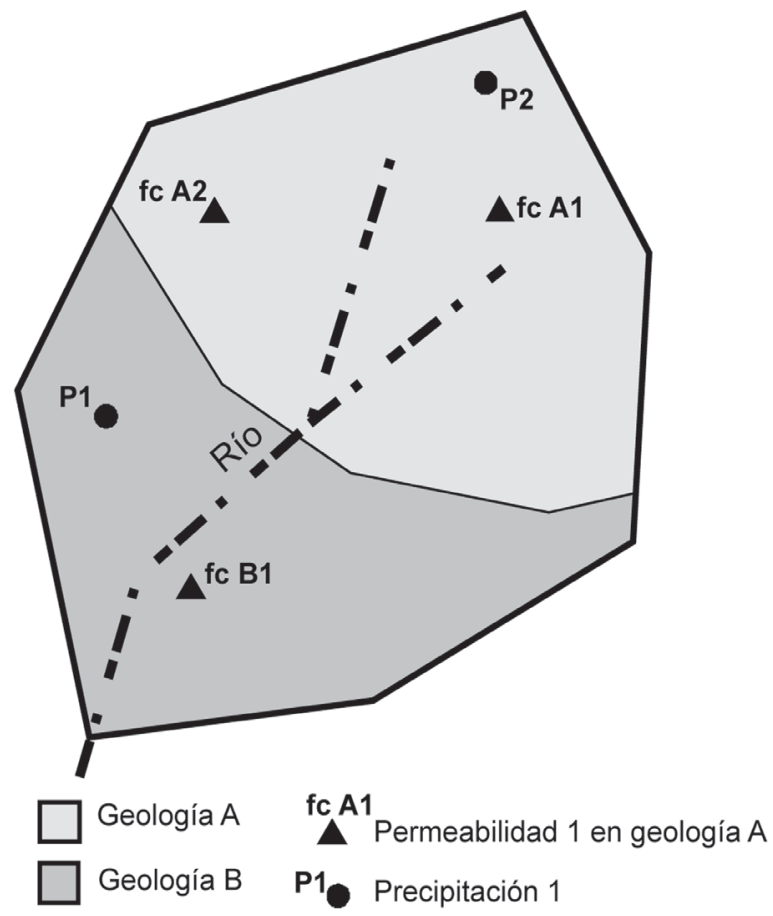

Fig. 1: Área de la cuenca, donde se muestra la geología A y B, tres pruebas de infiltración para obtener la permeabilidad del suelo y la precipitación de dos estaciones meteorológicas.

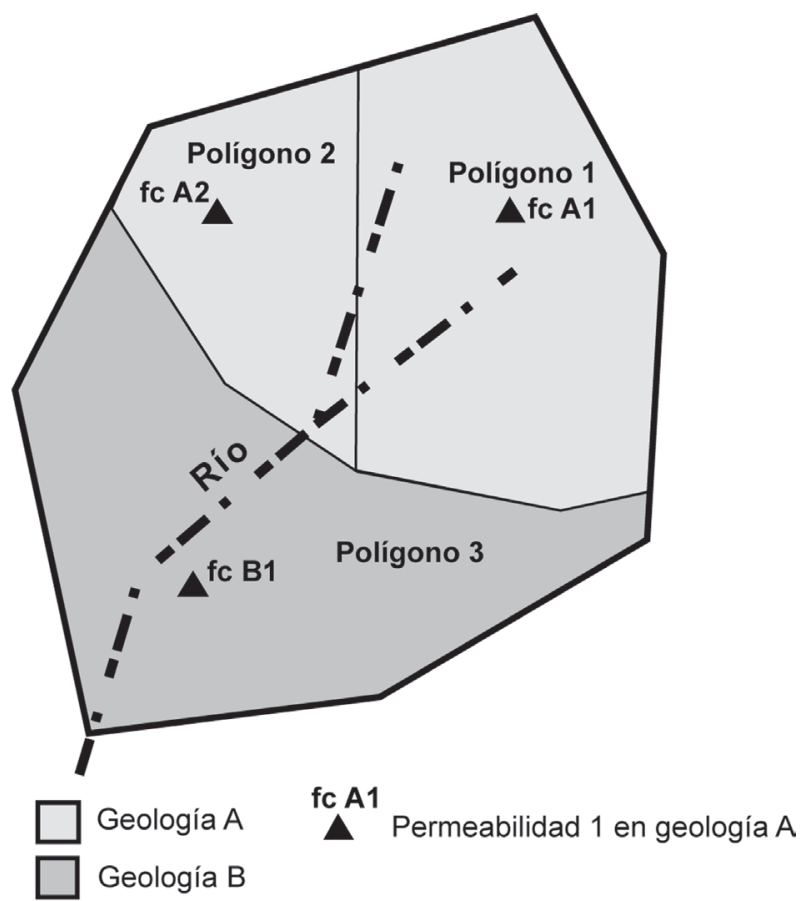

Fig. 2: Polígonos de Thiessen de cada prueba de infiltración dentro de cada una de las geologías. 


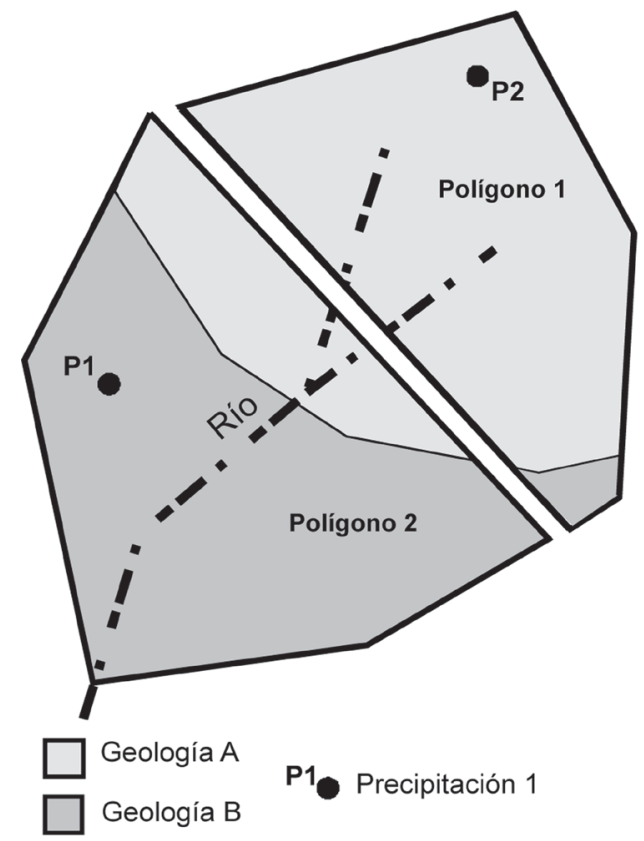

Fig. 3: Polígonos de Thiessen de cada estación meteorológica, dentro de la cuenca, para aplicar la precipitación correspondiente a cada polígono.

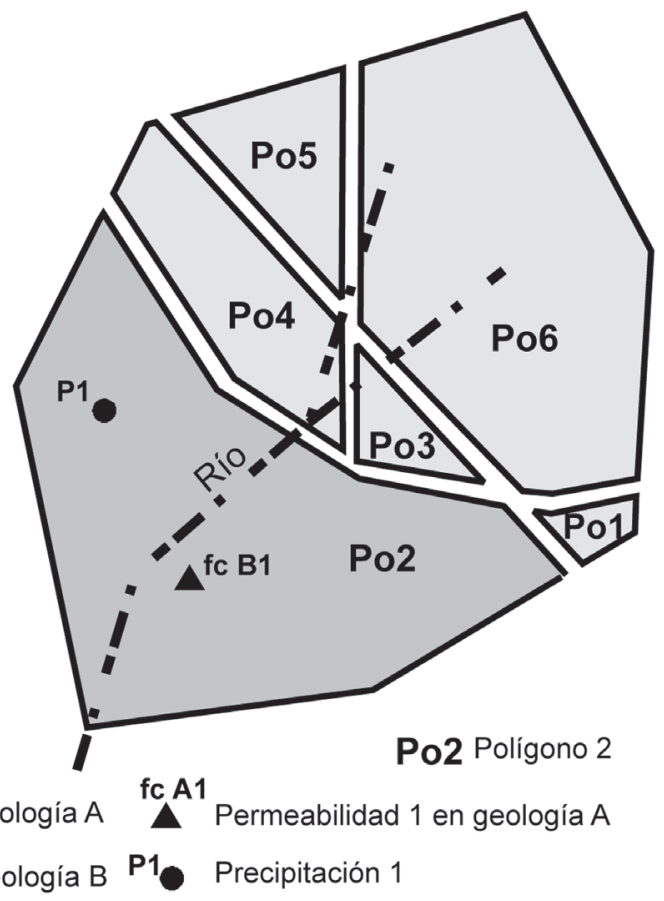

Fig. 4: Combinación de los polígonos de las figuras 2 y 3, de tal forma que cada polígono resultante tendrá su precipitación, geología y permeabilidad específica. 
Ejemplo 16 - Una cuenca está formada por dos polígonos. El primero con una recarga potencial de $200 \mathrm{~mm} / \mathrm{año}$ y un área de $6 \mathrm{~km}^{2}$. El segundo con una recarga potencial de $100 \mathrm{~mm} / \mathrm{año} \mathrm{y} \mathrm{un} \mathrm{área} \mathrm{de} 4 \mathrm{~km}^{2}$. Determine la recarga potencial del acuífero de la cuenca, en metros cúbicos por año.

Utilizando la ecuación (20)

Recarga polígono $1 ; \mathrm{V}=(200 / 1000)(6 * 1000 * 1000)=1,2 * 10^{6} \mathrm{~m}^{3} /$ año

Recarga polígono $2 ; \mathrm{V}=(100 / 1000)(4 * 1000 * 1000)=0,4 * 10^{6} \mathrm{~m}^{3} /$ año

Total $1,6^{*} 10^{6} \mathrm{~m}^{3} /$ año

La recarga potencial, del acuífero de la cuenca, es de $1,6^{*} 10^{6} \mathrm{~m}^{3} /$ año.

\section{Explotación de aguas subterráneas en una cuenca mediante pozos}

En una cuenca hidrológica, además de la recarga potencial por infiltración de la precipitación, puede darse el caso que la cuenca se recargue lateralmente mediante acuíferos que colinden con la cuenca, o también por recarga de los ríos. Lo mismo puede darse en el caso de la descarga de un acuífero, que además de los pozos, se descarga lateralmente o como flujo base de los ríos.

En la mayoría de los casos, la cuenca hidrológica es razonablemente congruente con la cuenca hidrogeológica (Walton, 1970); lo que quiere decir que la descarga o recarga lateral no se da. Si existe recarga o descarga lateral, estas deberán calcularse utilizando métodos hidrogeológicos; entre ellos, la ecuación de TIL, que se muestra a continuación.

$$
\mathrm{Q}=\mathrm{TiL}
$$

Donde:

$\mathrm{Q}\left[\mathrm{m}^{3} /\right.$ día $]=$ Caudal subterráneo que fluye por un tubo de flujo.

$\mathrm{T}\left[\mathrm{m}^{2 /}\right.$ día $]=$ Trasmisividad del acuífero.

$\mathrm{L}[\mathrm{m}]=$ Ancho del tubo de flujo.

$\mathrm{i}$ [adimensional $]=$ Gradiente del aguas subterránea en el sitio que se midió $\mathrm{L}$.

Referente a la recarga o descarga en los ríos, se puede realizar mediante aforos diferenciales realizados en el río, cuando no existe escorrentía superficial. La diferencia de flujo entre los dos puntos aforados, viene a representar la recarga o descarga de aguas subterráneas, siempre y cuando no existan aportes o extracciones de agua, en el tramo de río, entre los dos puntos de aforo.

En una cuenca no es conveniente extraer mediante pozos, un caudal mayor que la recarga potencial; además, se debe de dejar una cantidad de agua, para el flujo base de los ríos, considerando la biodiversidad existente y el caudal, como flujo base que se explota de los ríos. También, se ha de considerar el caudal que fluye de las nacientes que están siendo utilizadas como agua potable.

\section{REFERENCIAS}

BUTLER, S., 1957: Engineering Hydrology.- 356 págs. Prentice Hall Inc. EE.UU.

CALVO, J., 1999: Principios de hidrología forestal tropical.- 136 págs. Inst. Tecnol. Costa Rica, Esc. Ing. Forestal, Cartago, Costa Rica.

GRASSI, C., 1976: Resumen de propiedades físicas del suelo, métodos de riego.-265 págs. CIDIAT. Mérida, Venezuela.

HERAS, R., 1972: Manual de hidrología, Hidrología agrícola.- 330 págs: Esc. Hidrología, Madrid, España.

LINSLEY, R., KOHLER, M. \& PAULUS, L., 1958: Hydrology for engineers.- 340 págs. McGraw Hill, NuevaYork.

ONU, 1972: Manual de instrucciones, estudios hidrológicos.- 540 págs. ONU Publ. No 70, San José, Costa Rica.

SCHOSINSKY, G. \& LOSILLA, M., 2000: Modelo analítico para determinar la infiltración con base en la lluvia mensual.- Rev. Geol. Amér. Central, 23: 43-55.

WALTON, W, 1970: Ground water resource evaluation.- 664 págs. Mc Graw Hill, New York. 